\title{
A IMPORTÂNCIA DE PARQUES URBANOS PARA A CONSERVAÇÃO DE AVES
}

Ana Claudia de Almeida ${ }^{1}$

José Flávio Cândido Júnior ${ }^{2}$

\begin{abstract}
ALMEIDA, A. C. de; CÂNDIDO JÚNIOR, J. F. A importância de parques urbanos para a conservação de aves Arq. Ciênc.
\end{abstract} Vet. Zool. UNIPAR, Umuarama, v. 20, n. 4, p. 189-199, out./dez. 2017.

RESUMO: Devido à intensa modificação dos ambientes naturais e sua transformação em ambientes urbanos, muitas espécies da fauna silvestre têm perdido ambientes adequados à sua sobrevivência e reprodução. Algumas espécies de aves têm conseguido, entretanto, se adaptar a parques e praças urbanos, encontrando locais para reprodução, alimentação e abrigo. Este estudo avaliou o uso dos parques Tarquínio Joslin dos Santos, Danilo Galafassi, Paulo Gorski e Bosque Elias Lopuch, em Cascavel, PR, Brasil, por aves, e buscou compreender como se dá essa utilização por diferentes espécies. A primeira atividade realizada foi o levantamento das espécies de aves nos parques. A coleta dos dados foi feita por meio de reconhecimento visual e auditivo. Foi observado um total de 85 espécies: 63 espécies no Parque Tarquínio Joslin dos Santos, 48 espécies no Parque Danilo Galafassi, 62 espécies no Parque Municipal Paulo Gorski e 32 espécies no Bosque Elias Lopuch. Registros de nidificação, de insetivoria, nectarivoria e frugivoria, e de uso do espaço para abrigo permitiram inferir que os parques urbanos estudados podem ser importantes espaços para as aves e utilizados como parte de estratégias para sua conservação.

PALAVRAS-CHAVE: Ambientes naturais. Avifauna. Corredores urbanos. Levantamento. Urbanização.

\section{THE IMPORTANCE OF URBAN PARKS FOR BIRD PRESERVATION}

\begin{abstract}
Due to the intense modification in natural environments and their transformation in urban zones, many wildlife species have lost appropriate environments for their survival and reproduction. However, some bird species have been able to adapt to urban parks and squares, finding places for breeding, feeding and sheltering. This study evaluated the use of Tarquinio Joslin dos Santos, Danilo Galafassi, Paulo Gorski and Elias Lopuch parks located in the city of Cascavel, in the state of Paraná, Brazil, by birds, and sought to understand how different species use these resources. The first activity performed was the listing of the bird species sighted in the parks. A total of 85 species were observed: 63 species in Tarquínio Joslin dos Santos Park; 48 species in Danilo Galafassi Park; 62 species in Paulo Gorski Municipal Park; and 32 species in Elias Lopuch Park. The registration of nesting, insectivorous, nectarivorous and frugivorous activities, and the use of sheltering allowed implying that urban parks may be important areas for birds and may be used as part of the strategies for their preservation.

KEYWORDS: Avifauna. Natural environments. Surveys. Urban corridors. Urbanization.
\end{abstract}

\section{LA IMPORTANCIA DE BOSQUES URBANOS PARA CONSERVACIÓN DE AVES}

RESUMEN: Debido a la intensa modificación de los ambientes naturales y su transformación en ambientes urbanos, muchas especies de la fauna silvestre han perdido ambientes adecuados a su supervivencia y reproducción. Algunas especies de aves han conseguido adaptarse a parques y plazas, encontrando sitios para reproducción, alimentación y abrigo. Este estudio evaluó el uso de los parques Tarquínio Joslin dos Santos, Danilo Galafassi, Paulo Gorski y Bosque Elias Lopuch, en Cascavel - PR, Brasil, por aves, y ha buscado comprender cómo se da esa utilización por diferentes especies. La primera actividad realizada fue el levantamiento de las especies de aves en los bosques. La recolección de datos ha sido hecha a través de reconocimiento visual y auditivo. Se ha observado un total de 85 especies: 63 especies en el Parque Tarquínio Joslin dos Santos, 48 especies en el Parque Danilo Galafassi, 62 especies en el Parque Municipal Paulo Gorski y 32 especies en el Bosque Elias Lopuch. Registros de nidificación, de insectívora, nectarívora y frugívora, y de uso del espacio para abrigo, donde permitió inferir que los bosques urbanos estudiados pueden ser importantes espacios para las aves y utilizados como parte de estrategias para su conservación.

PALABRAS CLAVE: Ambientes naturales. Avifauna. Locales Urbanos. Levantamiento. Urbanización.

DOI: 10.25110 /arqvet.v20i4.2017.5476

${ }^{1}$ Filiação durante a pesquisa: Laboratório de Ecologia e Conservação, Universidade Estadual do Oeste do Paraná, Rua Universitária, 2069, Jardim Universitário, CEP 85819-110, Cascavel, PR, Brasil. Filiação atual: RPPN Buraco das Araras, Rodovia BR-267, km 510, Zona Rural, CEP 79240-000, Jardim, MS, Brasil. E-mail:monitoramentoararavermelha@gmail.com).

${ }^{2}$ Laboratório de Ecologia e Conservação, Universidade Estadual do Oeste do Paraná, Rua Universitária, 2069, Jardim Universitário, CEP 85819-110, Cascavel, PR, Brasil 


\section{Introdução}

O processo de urbanização e a retirada da vegetação natural para atividades humanas (em especial atividades agropecuárias) transformam profundamente os ambientes naturais, levando à fragmentação de habitats e afetando severamente a composição de diversas comunidades da fauna silvestre, inclusive da avifauna (TILMAN, 1999; MCKINNEY, 2002; CHATTERJEE; BASU, 2015). Sem uma matriz vegetal apropriada, muitas espécies silvestres de uma região têm dificuldade em obter abrigo (SEMPO; CANONGE; DENEUBOURG, 2013) e alimento, refletindo diretamente na sua capacidade reprodutiva e manutenção de populações minimamente viáveis (NASON; HAMRICK, 1997).

Frente à forte perturbação de seus ambientes naturais, os animais normalmente optam por deslocar-se para outras áreas menos impactadas ou adaptar-se às novas condições, minimizando assim os riscos de extinção. Essas duas possibilidades são muito comuns em aves e, enquanto algumas se valem da sua facilidade de deslocamento e buscam áreas mais adequadas à sua sobrevivência, outras se adaptam à convivência com o ser humano, uma tendência sinantrópica (VOSS, 1984).

O uso de áreas verdes no ambiente urbano é uma alternativa para a conservação de diversas espécies da fauna e flora. Guimarães (2006) menciona a importância de buscar por estratégias, nos centros urbanos, que possam permitir uma harmonia entre as exigências da vida humana e as necessidades ecológicas de outras espécies. Para isso, são essenciais áreas com cobertura vegetal arbórea, mesmo que sejam de pequenas dimensões, como parques urbanos, bosques, jardins, gramados e vias públicas arborizadas (ARGEL-DE-OLIVEIRA, 1995; ADLER; TANNER, 2015), estas últimas chamadas de corredores verdes urbanos (CVUs), utilizados para conectar praças, bairros e parques, e auxiliando na conservação da biodiversidade (PENTEADO; ALVAREZ, 2007). Dentre essas áreas, destacam-se os parques, que podem sustentar uma mistura de espécies urbanas e não urbanas de aves (ADLER; TANNER, 2015).

Visando a conservação das aves, o presente estudo foi realizado de forma a conhecer a diversidade da avifauna urbana de Cascavel, verificando quais recursos dos parques podem ser manejados a fim de estimular a permanência e/ ou reprodução de uma determinada espécie nesses locais, a passagem de aves migratórias, os aspectos de reprodução/ nidificação, alimentação e uso dos parques como locais de descanso pelas aves, e analisando as comunidades de cada parque quanto ao número de espécies, tamanho do parque e disponibilidade de alimento.

\section{Material e Métodos}

O estudo foi realizado nos parques Tarquínio Joslin dos Santos, Danilo Galafassi, Paulo Gorski e Bosque Elias Lopuch, em Cascavel, Paraná. O clima da região é definido como subtropical (Cfa), caracterizado por chuvas bem distribuídas durante o ano, verões quentes e invernos frios, e subclassificado como região temperada úmida de altitudes médias (LIMBERGER, 2007).

O Parque Tarquínio Joslin dos Santos apresenta uma área de aproximadamente $77.600 \mathrm{~m}^{2}$, com vegetação caracte- rizada por arbustos e árvores de até $30 \mathrm{~m}$ de altura. O Parque Municipal Danilo Galafassi possui uma área de $17.910 \mathrm{~m}^{2}$, com o objetivo de preservar as nascentes do rio Cascavel e conservar araucárias e outras árvores. Adjacente ao Parque Municipal Danilo Galafassi, encontra-se o Parque Municipal Paulo Gorski, conhecido como Lago Municipal, com uma área de $111.260 \mathrm{~m}^{2}$, sendo que $55.350 \mathrm{~m}^{2}$ são de mata nativa e $41.000 \mathrm{~m}^{2}$ de lâmina d'água (PORTAL DO MUNICÍPIO DE CASCAVEL, 2015). O Bosque Municipal Elias Lopuch se encontra no oeste da cidade, apresentando uma área de $8.177 \mathrm{~m}^{2}$ (PORTAL DO MUNICÍPIO DE CASCAVEL, 2017).

Foi realizado um levantamento das espécies, com anotações de características ecológicas das aves, como alimentação e uso dos espaços para abrigo e nidificação. As aves foram divididas em guildas alimentares de acordo com os hábitos alimentares observados. Os comportamentos relacionados à frugivoria foram divididos nas categorias "Modo de coleta", "Modo de ingestão", "Carregar no bico" e "Número de visitas", adaptadas de Argel-de-Oliveira, Castiglioni e Souza (1996) e Caetano (2005). A coleta dos dados foi realizada por meio de reconhecimento visual e auditivo, com auxílio de binóculos e guias ornitológicos. As observações foram desenvolvidas entre as $6 \mathrm{~h}$ e $10 \mathrm{~h}$ e entre as $16 \mathrm{~h}$ e $18 \mathrm{~h}$, no período de fevereiro de 2010 a julho de 2011, totalizando 93 amostragens e 186 horas.

As análises estatísticas foram realizadas utilizando-se o programa estatístico R (R CORE TEAM, 2013). A frequência de ocorrência (FO) das espécies foi calculada em cada parque, a partir da relação do número de registros de cada espécie pelo número total de visitas, classificando em categorias de ocupação adaptadas de Mendonça-Lima e Fontana (2000). As frequências dos diferentes hábitos alimentares foram comparadas por meio do teste do Qui-quadrado, testando-se a hipótese de que não há diferença entre as mesmas. $\mathrm{O}$ índice de similaridade de Jaccard foi utilizado para avaliar o grau de semelhança entre os parques estudados. Os critérios de avaliação para similaridade foram considerados segundo Drumond et al. (1982). O teste de homogeneidade foi utilizado para testar a hipótese de que as diferentes populações possuem a mesma proporção em todos os parques, onde foi verificado se o tamanho do parque e sua composição afetam o número de espécies. A análise de regressão linear foi utilizada para testar a hipótese de que o número de espécies não aumenta com o aumento do tamanho do parque. Foram calculadas as frequências de comportamentos em relação à frugivoria e utilizou-se o método do $\chi 2$ para verificar se estas diferiam entre si. Todos os testes foram interpretados utilizando-se nível de significância de 5\% $(\alpha=0,05)$.

\section{Resultados}

Foram registradas 85 espécies de aves nos quatro parques estudados: 63 espécies no Parque Tarquínio Joslin dos Santos, 48 espécies no Parque Danilo Galafassi, 62 espécies no Parque Municipal Paulo Gorski, e 32 espécies no Bosque Elias Lopuch (Tabela 1). Em todos os parques a ordem Passeriformes foi a mais representativa, com 48 espécies $(56,47 \%)$. 
Tabela 1: Lista de aves registrada nos parques estudados e suas frequências de ocorrência. A ordem taxonômica e nomenclatura seguem o Comitê Brasileiro de Registros Ornitológicos (CBRO; PIACENTINI et al., 2015). Áreas de estudo: 1 - Parque Tarquínio Joslin dos Santos; 2 - Parque Municipal Danilo Galafassi; 3 - Parque Paulo Gorski; 4 - Bosque Elias Lopuch.

\begin{tabular}{|c|c|c|c|c|c|c|}
\hline \multirow{2}{*}{ Nome científico } & \multirow{2}{*}{ Nome comum } & \multirow{2}{*}{$\begin{array}{c}\text { Hábito } \\
\text { alimentar } \\
\text { observado }\end{array}$} & \multicolumn{4}{|c|}{ Áreas de estudo } \\
\hline & & & 1 & 2 & 3 & 4 \\
\hline Amazonetta brasiliensis (Gmelin, 1789) & Ananaí & - & - & - & $\mathrm{O}$ & - \\
\hline Nannopterum brasilianus (Gmelin, 1789) & Biguá & PIS & $\mathrm{O}$ & $\mathrm{O}$ & $\mathrm{R}$ & - \\
\hline Nycticorax nycticorax (Linnaeus, 1758) & Socó-dorminhoco & PIS & - & - & $\mathrm{O}$ & $\mathrm{R}$ \\
\hline Butorides striata (Linnaeus, 1758) & Socozinho & PIS & $\mathrm{O}$ & $\mathrm{O}$ & $\mathrm{R}$ & - \\
\hline Egretta thula (Molina, 1782) & Garça-branca-pequena & INS & $\mathrm{O}$ & - & $\mathrm{R}$ & - \\
\hline Coragyps atratus (Bechstein, 1793) & Urubu & - & $\mathrm{O}$ & $\mathrm{R}$ & $\mathrm{P}$ & $\mathrm{O}$ \\
\hline Elanus leucurus (Vieillot, 1818) & Gavião-peneira & - & $\mathrm{O}$ & - & - & - \\
\hline Rupornis magnirostris (Gmelin, 1788) & Gavião-carijó & CAR & $\mathrm{P}$ & $\mathrm{P}$ & $\mathrm{P}$ & - \\
\hline Aramides saracura (Spix, 1825) & Saracura-do-mato & - & $\mathrm{O}$ & $\mathrm{O}$ & $\mathrm{O}$ & - \\
\hline Gallinula galeata (Lichtenstein, 1818) & Galinha-d'água & INS & - & - & $\mathrm{P}$ & - \\
\hline Vanellus chilensis (Molina, 1782) & Quero-quero & INS & $\mathrm{R}$ & $\mathrm{O}$ & $\mathrm{R}$ & $\mathrm{P}$ \\
\hline Jacana jacana (Linnaeus, 1766) & Jaçanã & INS & $\mathrm{O}$ & - & $\mathrm{O}$ & - \\
\hline Columbina minuta (Linnaeus, 1766) & Rolinha-de-asa-canela & GRA & $\mathrm{O}$ & $\mathrm{O}$ & - & - \\
\hline Columbina talpacoti (Temminck, 1811) & Rolinha & GRA & $\mathrm{O}$ & $\mathrm{O}$ & - & - \\
\hline Columba livia (Gmelin, 1789) & Pombo-doméstico & GRA & - & $\mathrm{R}$ & - & - \\
\hline Patagioenas picazuro (Temminck, 1813) & Asa-branca & GRA & $\mathrm{P}$ & $\mathrm{R}$ & $\mathrm{R}$ & $\mathrm{R}$ \\
\hline Zenaida auriculata (Des Murs, 1847) & Avoante & GRA & $\mathrm{R}$ & $\mathrm{R}$ & $\mathrm{R}$ & $\mathrm{R}$ \\
\hline Piaya cayana (Linnaeus, 1766) & Alma-de-gato & - & $\mathrm{O}$ & $\mathrm{P}$ & $\mathrm{P}$ & $\mathrm{O}$ \\
\hline Crotophaga ani (Linnaeus, 1758) & Anu-preto & INS & $\mathrm{P}$ & $\mathrm{P}$ & $\mathrm{R}$ & - \\
\hline Guira guira (Gmelin, 1788) & Anu-branco & INS & $\mathrm{P}$ & $\mathrm{P}$ & $\mathrm{P}$ & $\mathrm{O}$ \\
\hline Megascops choliba (Vieillot, 1817) & Corujinha-do-mato & INS & $\mathrm{P}$ & - & - & $\mathrm{O}$ \\
\hline Eupetomena macroura (Gmelin, 1788) & Beija-flor-tesoura & - & $\mathrm{O}$ & - & - & - \\
\hline Florisuga fusca (Vieillot, 1817) & Beija-flor-preto & - & $\mathrm{O}$ & - & - & - \\
\hline Anthracothorax nigricollis (Vieilott, 1817) & Beija-flor-de-veste-preta & - & $\mathrm{O}$ & - & - & $\mathrm{O}$ \\
\hline Chlorostilbon lucidus (Shaw, 1812) & Besourinho-de-bico-vermelho & NEC/INS & $\mathrm{P}$ & $\mathrm{P}$ & $\mathrm{P}$ & $\mathrm{P}$ \\
\hline Hylocharis chrysura (Shaw, 1812) & Beija-flor-dourado & NEC/INS & $\mathrm{R}$ & $\mathrm{P}$ & $\mathrm{P}$ & $\mathrm{P}$ \\
\hline Leucochloris albicollis (Vieillot, 1818) & Beija-flor-de-papo-branco & - & $\mathrm{O}$ & - & $\mathrm{O}$ & - \\
\hline Megaceryle torquata (Linnaeus, 1766) & Martim-percador-grande & PIS & $\mathrm{O}$ & - & $\mathrm{P}$ & - \\
\hline Chloroceryle amazona (Latham, 1790) & Martim-percador-verde & PIS & $\mathrm{O}$ & $\mathrm{O}$ & $\mathrm{P}$ & - \\
\hline Chloroceryle americana (Gmelin, 1788) & Martim-pescador-pequeno & PIS & $\mathrm{P}$ & - & $\mathrm{P}$ & - \\
\hline Pteroglossus castanotis (Gould, 1834) & Araçari-castanho & - & $\mathrm{O}$ & - & $\mathrm{O}$ & $\mathrm{O}$ \\
\hline Veniliornis spilogaster (Wagler, 1827) & Picapauzinho-verde-carijó & INS & $\mathrm{O}$ & $\mathrm{P}$ & - & $\mathrm{O}$ \\
\hline Colaptes melanochloros (Gmelin, 1788) & Pica-pau-verde-barrado & INS & $\mathrm{O}$ & $\mathrm{P}$ & - & $\mathrm{O}$ \\
\hline Colaptes campestris (Vieillot, 1818) & Pica-pau-do-campo & INS & - & - & $\mathrm{O}$ & - \\
\hline Caracara plancus (Miller, 1777) & Carcará & - & - & - & $\mathrm{P}$ & $\mathrm{O}$ \\
\hline Milvago chimachima (Vieillot, 1816) & Carrapateiro & - & $\mathrm{O}$ & - & - & - \\
\hline $\begin{array}{l}\text { Psittacara leucophthalmus (Statius Muller, } \\
\text { 1776) }\end{array}$ & Piriquitão-maracanã & FRU & - & - & - & $\mathrm{O}$ \\
\hline Furnarius rufus (Gmelin, 1788) & João-de-barro & INS & $\mathrm{R}$ & $\mathrm{R}$ & $\mathrm{R}$ & $\mathrm{R}$ \\
\hline Lochmias nematura (Lichtenstein, 1823) & João-porca & INS & $\mathrm{P}$ & $\mathrm{O}$ & - & - \\
\hline Leptasthenura setaria (Temminck, 1824) & Grimpeiro & INS & $\mathrm{O}$ & - & $\mathrm{O}$ & - \\
\hline Tolmomyias sulphurescens (Spix, 1825) & Bico-chato-de-orelha-preta & - & - & $\mathrm{O}$ & - & - \\
\hline Todirostrum cinereum (Linnaeus, 1766) & Ferreirinho-relógio & - & $\mathrm{O}$ & - & - & - \\
\hline Pachyramphus polychopterus (Vieillot, 1818) & Caneleiro-preto & - & - & - & $\mathrm{O}$ & - \\
\hline
\end{tabular}


Euscarthmus meloryphus (Wied, 1831)

Camptostoma obsoletum (Temminck, 1824)

Elaenia flavogaster (Thunberg, 1822)

Serpophaga subcristata (Vieillot, 1817)

Pitangus sulphuratus (Linnaeus, 1766)

Megarynchus pitangua (Linnaeus, 1766)

Myiozetetes similis (Spix, 1825)

Tyrannus melancholicus (Vieillot, 1819)

Tyrannus savana (Vieillot, 1808)

Empidonomus varius (Vieillot, 1818)

Hylophilus poicilotis Temminck, 1822

Cyanocorax chrysops (Vieillot, 1818)

Pygochelidon cyanoleuca (Vieillot, 1817)

Progne chalybea (Gmelin, 1789)

Troglodytes musculus (Naumann, 1823)

Turdus leucomelas (Vieillot, 1818)

Turdus rufiventris (Vieillot, 1818)

Turdus amaurochalinus (Cabanis, 1850)

Mimus saturninus (Lichtenstein, 1823)

Zonotrichia capensis (Statius Muller, 1776)

Setophaga pitiayumi (Vieillot, 1817)

Geothlypis aequinoctialis (Gmelin, 1789)

Basileuterus culicivorus (Deppe, 1830)

Myiothlypis leucoblephara (Vieillot, 1817)

Cacicus haemorrhous (Linnaeus, 1766)

Molothrus bonariensis (Gmelin, 1789)

Sturnella superciliaris (Bonaparte, 1850)

Pipraeidea bonariensis (Gmelin, 1789)

Tangara sayaca (Linnaeus, 1766)

Sicalis flaveola (Linnaeus, 1766)

Volatinia jacarina (Linnaeus, 1766)

Tachyphonus coronatus (Vieillot, 1822)

Tersina viridis (Illiger, 1811)

Dacnis cayana (Linnaeus, 1766)

Sporophila lineola (Linnaeus, 1758)

Sporophila plumbea (Wied, 1830)

Sporophila caerulescens (Vieillot, 1823)

Saltator similis (d'Orbigny \& Lafresnaye, 1837)

Pyrrhocoma ruficeps (Strickland, 1844)

Euphonia chlorotica (Linnaeus, 1766)

Chlorophonia cyanea (Thunberg, 1822)

Passer domesticus (Linnaeus, 1758)

Barulhento

Risadinha

Guaracava-de-barriga-amarela

Alegrinho

Bem-te-vi

Neinei

Bentivizinho-de-penachovermelho

$\begin{array}{lc}\text { Suiriri } & \text { INS } \\ \text { Tesourinha } & \text { FRU } \\ \text { Peitica } & \text { INS } \\ \text { Verdinho-coroado } & -\end{array}$

Gralha-picaça

Andorinha-pequena-de-casa

Andorinha-grande

Corruíra

Sabiá-branco

Sabiá-laranjeira

Sabiá-poca

Sabiá-do-campo

Tico-tico

Mariquita

Pia-cobra

Pula-pula

Pula-pula-assobiador

Guaxe

Chupim

Polícia-inglesa-do-sul

Sanhaço-papa-laranja

Sanhaçu-cinzento

Canário-da-terra

Tiziu

Tiê-preto

Saí-andorinha

Saí-azul

Bigodinho

Patativa

Colerinho

Trinca-ferro-verdadeiro

Cabecinha-castanha

Fim-fim

Gaturamo-bandeira

Pardal

INS/GRA $\quad \mathrm{P} \quad \mathrm{R} \quad \mathrm{R} \quad \mathrm{R}$

Legenda: Hábitos alimentares observados: PIS - piscívoro; INS - insetívoro; CAR - carnívoro; GRA - granívoro; FRU - frugívoro; NEC - nectarívoro; ONI - onívoro. Áreas do estudo: 1 - Parque Tarquínio Joslin dos Santos; 2 - Parque Municipal Danilo Galafassi; 3 - Parque Municipal Paulo Gorski; 4 - Bosque Elias Lopuch. FO: R - residente; P - provável residente; O - ocasional e/ou sobrevoante. 
Os parques apresentaram similaridade média em relação à sua composição avifaunística (Figura 1 e Tabela $2)$. Houve uma diferença significativa no número total de espécies entre os parques $(\chi 2=12,385 ; \mathrm{p}<0,01)$. A análise de regressão linear mostrou diferença estatística $(\mathrm{t}=5,519 ; \mathrm{p}=$ $0,031)$, indicando que quanto maior o tamanho do parque, maior o número de espécies de aves encontradas (Figura 2).

Figura 1: Dendograma da similaridade entre os parques (Índice de Jaccard).

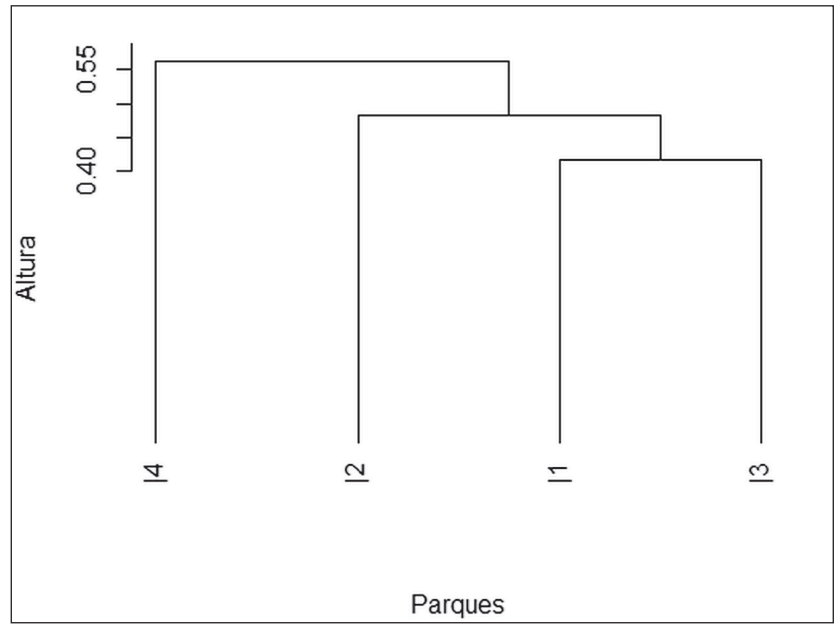

Legenda: 1 - Parque Tarquínio Joslin dos Santos; 2 - Parque Danilo Galafassi; 3 - Parque Municipal Paulo Gorski; 4 - Bosque Elias Lopuch.

Tabela 2: Índices de similaridade (Jaccard) calculados entre os parques estudados em Cascavel, PR.

\begin{tabular}{lccc}
\hline & $\begin{array}{c}\text { Danilo } \\
\text { Galafassi }\end{array}$ & Tarquínio & $\begin{array}{c}\text { Elias } \\
\text { Lopuch }\end{array}$ \\
\hline Paulo Gorski & 0,53 & 0,58 & 0,40 \\
$\begin{array}{l}\text { Danilo } \\
\text { Galafassi }\end{array}$ & - & 0,52 & 0,45 \\
Tarquínio & - & - & 0,46 \\
\hline
\end{tabular}

Muitas espécies tiveram registros pouco frequentes, e foram categorizadas como ocasionais e/ou sobrevoantes (Tabela 1). A espécie mais comum nos parques é a Zenaida auriculata $(\mathrm{FO}=100 \%)$, com população que pode exceder centenas de indivíduos nos Parques Tarquínio Joslin dos Santos, Paulo Gorski e Danilo Galafassi. Sua presença em toda região se deve, principalmente, à intensa atividade agrícola do oeste do Paraná, que produz ampla oferta de grãos durante todo o ano. As espécies Columba livia, Furnarius rufus, Turdus amaurochalinus, Turdus rufiventris, Turdus leucomelas, Pitangus sulphuratus e Passer domesticus também apresentaram altas frequências de ocorrência nos parques onde foram observadas. A espécie Sporophila plumbea (patativa) foi registrada em apenas dois parques, sendo ocasional no Parque Tarquínio e provável residente no Parque Paulo Gorski, também registrada por Model, Remor e Nascimento (2014) nesses locais, sugerindo a importância desses parques para a conservação dessa espécie, visto que, apesar de seu status ser considerado pouco preocupante (LC) pela IUCN (IUCN, 2015), no estado do Paraná ela é considerada ameaçada de extinção devido ao comércio ilegal e destruição de florestas
(IAP, 2006).

Figura 2: Correlação linear entre o número de espécies de aves registradas em cada parque e o tamanho do parque.

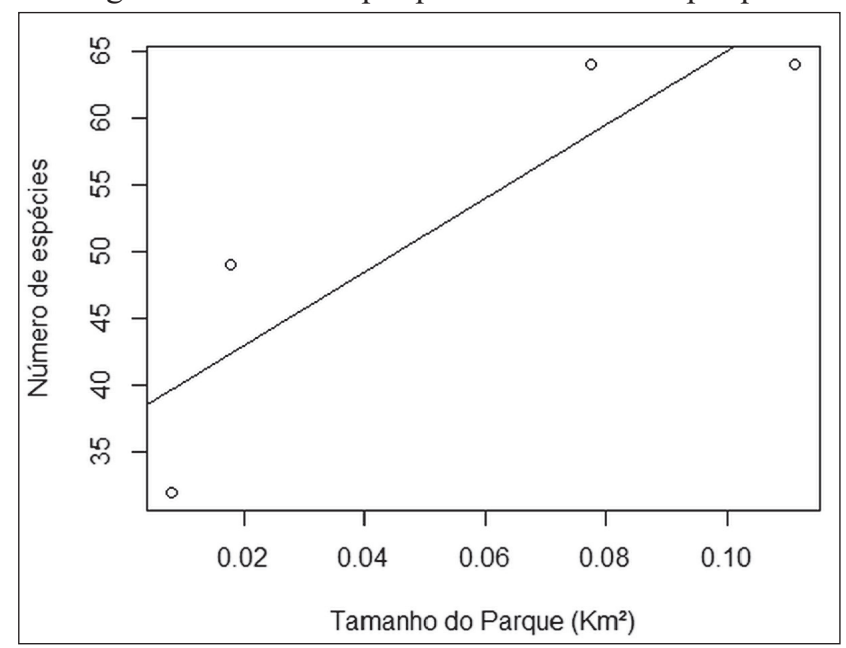

Reprodução

Durante o período observado, foram confirmadas atividades reprodutivas (construção de ninhos, acasalamento e cuidado parental) de Zenaida auriculata, Furnarius rufus, e Turdus amaurochalinus e Tyrannus savana no Parque Tarquínio, Zenaida auriculata e Cacicus haemorrhous no Parque Danilo Galafassi, Vanellus chilensis, Zenaida auriculata, Pitangus sulphuratus e Tyrannus melancholicus no Parque Paulo Gorski, Zenaida auriculata, Furnarius rufus, Turdus leucomelas e Passer domesticus no Bosque Elias Lopuch. No Parque Municipal Danilo Galafassi foram observados em torno de 25 ninhos da espécie Cacicus haemorrhous em um indivíduo de Araucaria angustifolia. Outras espécies, embora não tenha sido confirmada a reprodução, permaneceram formando casais durante o período observado, como Megascops choliba, Chlorostilbon lucidus, Chloroceryle americana, Euphonia chlorotica, Tersina viridis e Volatinia jacarina. Foi registrada também, a presença de indivíduos juvenis das espécies Milvago chimachima, Rupornis magnirostris e Guira guira.

\section{Alimentação}

A maioria das espécies observadas utiliza o parque também como local para obtenção de alimento. Foi registrado o comportamento de alimentação de 57 espécies de aves (Tabela 1). Os hábitos alimentares predominantes foram: insetívoro $(36,21 \%)$, granívoro $(22,41 \%)$ e frugívoro $(13,79 \%)$, apresentando diferença significativa $(\chi 2=118,243 ; \mathrm{p}<0,01)$ (Figura 3). 
Figura 3: Hábitos alimentares apresentadas pelas aves presentes nos diferentes parques.

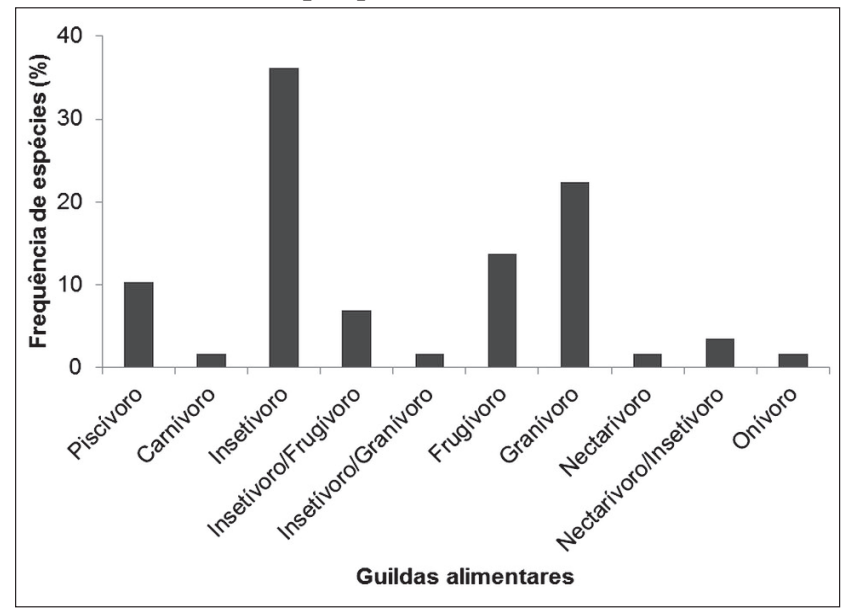

Os parques possuem grande oferta de insetos para o consumo pelas aves insetívoras, principalmente no verão, período com maior quantidade de chuva. Foram encontrados regurgitos de Megascops choliba contendo exoesqueleto de indivíduos da família Formicidae. Macroinvertebrados aquáticos são encontrados em grande quantidade nos parques Tarquínio e Paulo Gorski, sendo consumidos por Egretta thula, Jacana jacana, Butorides striata, Vanellus chilensis, Gallinula galeata, Lochmias nematura e Furnarius rufus, além do parque fornecer peixes para a alimentação de Phalacrocorax brasilianus, Chloroceryle americana, Chloroceryle amazona e Megaceryle torquata.

A nectarivoria foi registrada em algumas espécies de plantas por beija-flores: Chlorostilbon lucidus foi observado se alimentando em Podocarpus lambertii, Impatiens walleriana, Chorisia speciosa, Ipomoeapurpurea, Jacaranda mimosifolia e Ludwigia sp.; Hylocharis chrysura foi observado se alimentando em Bauhinia variegata, Hibiscus rosa-sinensise Sanchezia speciosa; e Leucochloris albicollis foi observado se alimentando em Bauhinia variegata. Essas visitas sugerem que a polinização possa ser realizada pelos mesmos, exceto em Jacaranda mimosifolia, na qual C. luci$d u s$ atuou como um pilhador de néctar.Também foi registrada frugivoria em diversas espécies de plantas (Tabela 3).

Tabela 3: Espécies de plantas cujos frutos foram consumidos pelas aves em cada parque estudado.

\begin{tabular}{|c|c|c|}
\hline Espécie de planta & Nome comum & Parque com registro de frugivoria \\
\hline Syagrus romanzoffiana (Cham.) Glassman & Jerivá & Bosque Municipal Elias Lopuch \\
\hline Ilex paraguariensis A.St.-Hil. & Erva-mate & Parque Tarquínio Joslin dos Santos \\
\hline Blepharocalyx salicifolius (Kunth) O. Berg & Cambuí & Parque Tarquínio Joslin dos Santos \\
\hline Ficus calyptroceras (Miq.) Miq. & Figueira & Parque Municipal Danilo Galafassi \\
\hline Schinus terebinthifolius Raddi & Aroeira-vermelha & Parque Municipal Paulo Gorski \\
\hline Cupania vernalis Camb. & Camboatá-vermelho & $\begin{array}{l}\text { Bosque Municipal Elias Lopuch e Parque Municipal } \\
\text { Paulo Gorski }\end{array}$ \\
\hline Nectandra rígida (Kunth) Nees & Canela-ferrugem & Parque Tarquínio Joslin dos Santos \\
\hline Ocotea pulchella Mart. & Canela-lageana & $\begin{array}{l}\text { Bosque Municipal Elias Lopuch e Parque Tarquínio } \\
\text { Joslin dos Santos }\end{array}$ \\
\hline Trema micrantha (L.) Blume & Grandiúva & Parque Municipal Danilo Galafassi \\
\hline Ligustrum lucidus W. T. Aiton & Ligustro & Parque Municipal Paulo Gorski \\
\hline
\end{tabular}

Os comportamentos frugívoros são mostrados nas Tabelas 4-7. Em relação ao modo de coleta, a maioria das espécies observadas coletou em pouso, representando $87,9 \%$, enquanto as coletas durante o voo representaram $12,1 \%$, diferindo significativamente $(\chi 2=57,456 ; p<0,01)$. Analisando-se o modo de ingestão, $51,6 \%$ da ingestão foram do fruto inteiro e $48,4 \%$ de parte do fruto, sem diferença significativa $(\chi 2=0,102 ; p=0,75)$. A categoria "Carregar no bico" se refere à coleta do fruto seguido de voo para longe da planta carregando o fruto no bico. O comportamento de carregar no bico foi menos frequente $(20,7 \%)$ do que não carregar no bico $(79,3 \%)$, diferindo significativamente $(\chi 2=34,339$; $\mathrm{p}<0,01)$. O número de visitas à planta foi dividido em "uma visita" (13,8\%), "poucas visitas" $(27,6 \%)$ e "muitas visitas" $(58,6 \%)$, com diferença significativa $(\chi 2=31,585 ; \mathrm{p}<0,01)$.

Tabela 4: Modo de coleta dos frutos pelas aves.

\begin{tabular}{|c|c|c|c|c|c|c|c|c|c|c|}
\hline Espécie de ave & 1 & 2 & 3 & 4 & 5 & 6 & 7 & 8 & 9 & 10 \\
\hline P. leucophthalmus & $\mathrm{p}$ & - & - & - & - & - & - & - & - & - \\
\hline P. sulphuratus & - & $\mathrm{p} / \mathrm{v}$ & $\mathrm{p}$ & $\mathrm{p}$ & - & $\mathrm{p}$ & - & - & - & - \\
\hline T. savana & - & - & $\mathrm{p} / \mathrm{v}$ & - & - & $\mathrm{p} / \mathrm{v}$ & - & - & - & - \\
\hline T. leucomelas & - & - & - & - & - & $\mathrm{p}$ & $\mathrm{p}$ & - & - & - \\
\hline T. rufiventris & - & - & $\mathrm{p}$ & $\mathrm{p}$ & - & - & - & - & - & - \\
\hline T. amaurochalinus & - & - & $\mathrm{p} / \mathrm{v}$ & $\mathrm{p}$ & - & $\mathrm{p}$ & $\mathrm{p}$ & $\mathrm{p}$ & - & - \\
\hline T. coronatus & - & - & - & - & - & - & - & - & $\mathrm{p}$ & - \\
\hline
\end{tabular}




\begin{tabular}{|c|c|c|c|c|c|c|c|c|c|c|}
\hline T. sayaca & - & $\mathrm{p}$ & $\mathrm{p}$ & $\mathrm{p}$ & $\mathrm{p}$ & - & - & $\mathrm{p}$ & - & - \\
\hline T. viridis & - & - & - & - & - & - & $\mathrm{p}$ & - & - & - \\
\hline S. caerulescens & - & - & - & $\mathrm{p}$ & - & - & - & - & - & $\mathrm{p}$ \\
\hline C. haemorrhous & - & - & - & - & - & $\mathrm{p}$ & - & - & - & - \\
\hline M. bonariensis & - & - & - & - & - & $\mathrm{p}$ & - & - & - & - \\
\hline E. chlorotica & - & - & $\mathrm{p}$ & - & - & - & - & $\mathrm{p}$ & - & - \\
\hline
\end{tabular}

Legenda: Modo de coleta: $\mathrm{p}$ = aves que coletaram frutos em pouso; $\mathrm{v}=$ aves que coletaram frutos em voo. Plantas: 1: Syagrus romanzoffiana; 2: Ilex paraguariensis; 3: Blepharocalyx salicifolius; 4: Ficus calyptroceras; 5: Schinus terebinthifolius; 6: Cupania vernalis; 7: Nectandra rigida; 8: Ocotea pulchella; 9: Trema micrantha; 10: Ligustrum lucidus.

Tabela 5: Modo de ingestão dos frutos pelas aves.

\begin{tabular}{|c|c|c|c|c|c|c|c|c|c|c|}
\hline Espécie de ave & 1 & 2 & 3 & 4 & 5 & 6 & 7 & 8 & 9 & 10 \\
\hline p. leucophthalmus & $\mathrm{a}$ & - & - & - & - & - & - & - & - & - \\
\hline p. sulphuratus & - & $\mathrm{i}$ & $\mathrm{i}$ & $\mathrm{i} / \mathrm{a}$ & - & $\mathrm{a}$ & - & - & - & - \\
\hline t. savana & - & - & $\mathrm{i}$ & - & - & $\mathrm{a}$ & - & - & - & - \\
\hline t. leucomelas & - & - & - & - & - & $\mathrm{a}$ & a & - & - & - \\
\hline t. rufiventris & - & - & $\mathrm{i}$ & $\mathrm{i}$ & - & - & - & - & - & - \\
\hline t. amaurochalinus & - & - & $\mathrm{i}$ & $\mathrm{i}$ & - & $\mathrm{a}$ & $\mathrm{i}$ & $\mathrm{i}$ & - & - \\
\hline t. coronatus & - & - & - & - & - & - & - & - & $\mathrm{i}$ & - \\
\hline t. sayaca & - & $\mathrm{a}$ & $\mathrm{i}$ & $\mathrm{a}$ & $\mathrm{i} / \mathrm{a}$ & - & - & $\mathrm{i}$ & - & - \\
\hline t. viridis & - & - & - & - & - & - & $\mathrm{a}$ & - & - & - \\
\hline s. caerulescens & - & - & - & $\mathrm{a}$ & - & - & - & - & - & $\mathrm{i}$ \\
\hline c. haemorrhous & - & - & - & - & - & $\mathrm{a}$ & - & - & - & - \\
\hline m. bonariensis & - & - & - & - & - & $\mathrm{a}$ & - & - & - & - \\
\hline e. chlorotica & - & - & $\mathrm{i}$ & - & - & - & - & $\mathrm{a}$ & - & - \\
\hline
\end{tabular}

Legenda: Modo de ingestão: $\mathrm{i}$ = aves que engoliram o fruto inteiro; a = aves que engoliram parte do fruto. Plantas: 1: Syagrus romanzoffiana; 2: Ilex paraguariensis; 3: Blepharocalyx salicifolius; 4: Ficus calyptroceras; 5: Schinus terebinthifolius; 6: Cupania vernalis; 7: Nectandra rigida; 8: Ocotea pulchella; 9: Trema micrantha; 10: Ligustrum lucidus.

Tabela 6: Comportamento de carregar o fruto no bico.

\begin{tabular}{|c|c|c|c|c|c|c|c|c|c|c|}
\hline espécie de ave & 1 & 2 & 3 & 4 & 5 & 6 & 7 & 8 & 9 & 10 \\
\hline P. leucophthalmus & $\mathrm{n}$ & - & - & - & - & - & - & - & - & - \\
\hline P. sulphuratus & - & $\mathrm{n}$ & $\mathrm{n}$ & $\mathrm{n}$ & - & $\mathrm{n}$ & - & - & - & - \\
\hline T. savana & - & - & $\mathrm{s}$ & - & - & $\mathrm{n}$ & - & - & - & - \\
\hline T. leucomelas & - & - & - & - & - & $\mathrm{s}$ & $\mathrm{n}$ & - & - & - \\
\hline T. rufiventris & - & - & $\mathrm{n}$ & $\mathrm{n}$ & - & - & - & - & - & - \\
\hline T. amaurochalinus & - & - & $\mathrm{n}$ & $\mathrm{n}$ & - & $\mathrm{s}$ & $\mathrm{n}$ & $\mathrm{n}$ & - & - \\
\hline T. coronatus & - & - & - & - & - & - & - & - & $\mathrm{n}$ & - \\
\hline T. sayaca & - & $\mathrm{n}$ & $\mathrm{s}$ & $\mathrm{n}$ & $\mathrm{n}$ & - & - & $\mathrm{n}$ & - & - \\
\hline T. viridis & - & - & - & - & - & - & $\mathrm{s}$ & - & - & - \\
\hline S. caerulescens & - & - & - & $\mathrm{n}$ & - & - & - & - & - & $\mathrm{n}$ \\
\hline C. haemorrhous & - & - & - & - & - & $\mathrm{n}$ & - & - & - & - \\
\hline M. bonariensis & - & - & - & - & - & $\mathrm{n}$ & - & - & - & - \\
\hline E. chlorotica & - & - & $\mathrm{s}$ & - & - & - & - & $\mathrm{n}$ & - & - \\
\hline \multicolumn{11}{|c|}{$\begin{array}{l}\text { Legenda: Carregar no bico: } \mathrm{s}=\text { aves que carregaram } \\
\text { manzoffiana; } 2: \text { Ilex paraguariensis; } 3: \text { Blepharocalyx } \\
\text { 7: Nectandra rigida; } 8: \text { Ocotea pulchella; } 9: \text { Trema m }\end{array}$} \\
\hline Espécie de ave & 1 & 2 & 3 & 4 & 5 & 6 & 7 & 8 & 9 & 10 \\
\hline P. leucophthalmus & $\mathrm{M}$ & - & - & - & - & - & - & - & - & - \\
\hline P. sulphuratus & - & M & M & M & - & 1 & - & - & - & - \\
\hline
\end{tabular}




\begin{tabular}{|c|c|c|c|c|c|c|c|c|c|c|}
\hline T. savana & - & - & $\mathrm{V}$ & - & - & $\mathrm{M}$ & - & - & - & - \\
\hline T. leucomelas & - & - & - & - & - & $\mathrm{M}$ & $\mathrm{P}$ & - & - & - \\
\hline T. rufiventris & - & - & $\mathrm{V}$ & M & - & - & - & - & - & - \\
\hline T. amaurochalinus & - & - & M & M & - & M & $\mathrm{P}$ & $\mathrm{P}$ & - & - \\
\hline T. coronatus & - & - & - & - & - & - & - & - & 1 & - \\
\hline T. sayaca & - & 1 & V & M & $\mathrm{P}$ & - & - & M & - & - \\
\hline T. viridis & - & - & - & - & - & - & $\mathrm{P}$ & - & - & - \\
\hline S. caerulescens & - & - & - & M & - & - & - & - & - & $\mathrm{P}$ \\
\hline C. haemorrhous & - & - & - & - & - & $\mathrm{P}$ & - & - & - & - \\
\hline M. bonariensis & - & - & - & - & - & 1 & - & - & - & - \\
\hline E. chlorotica & - & - & $\mathrm{P}$ & - & - & - & - & $\mathrm{M}$ & - & - \\
\hline
\end{tabular}

Legenda: Número de visitas: 1 = uma visita; $\mathrm{P}$ = poucas visitas; $\mathrm{M}=$ muitas visitas. Plantas: 1:Syagrus romanzoffiana; 2: Ilex paraguariensis; 3: Blepharocalyx salicifolius; 4: Ficus calyptroceras; 5: Schinus terebinthifolius; 6: Cupania vernalis; 7: Nectandra rigida; 8: Ocotea pulchella; 9: Trema micrantha; 10: Ligustrum lucidus.

\section{Discussão}

A similaridade média entre os parques em relação à sua composição faunística pode ser explicada pelo grande número de espécies generalistas que compõem a maior parte da avifauna dos parques, o que permite a exploração de diferentes ambientes pelas mesmas (VALADÃO; FRANCHIN; MARÇAL JR., 2006).

A diferença no número de espécies entre os parques pode ser explicada pelo tamanho do parque, a estrutura e a composição da vegetação do mesmo. Hábitats mais complexos tendem a sofrer variações menos acentuadas de recursos, mantendo a diversidade de sua avifauna constante (MOTTA-JÚNIOR,1990), e praças maiores, com vegetação variada e que conservem parte da vegetação natural são importantes para a manutenção de uma avifauna diversificada (MATARAZZO-NEUBERGER,1995). Os parques Paulo Gorski, Danilo Galafassi e Tarquínio Joslin dos Santos apresentam uma grande área com diversificação de hábitat e, principalmente este último, de vegetação que serve como alimento, fornecendo suporte para a manutenção de um número maior de espécies quando comparado ao Bosque Elias Lopuch. Além disso, a diversidade de espécies é significativamente maior em áreas urbanas onde há a manutenção de árvores adultas, ou seja, de árvores presentes por um longo período no local, sendo que elas são muito utilizadas para descanso, forrageio e nidificação pelas aves (BARTH; FITZGIBBON; WILSON, 2015).

Dentre as espécies registradas (Tabela 1), podem ser consideradas sinantrópicas Columba livia, Zenaida auriculata, Furnarius rufus, Turdus amaurochalinus, Turdus rufiventris, Turdus leucomelas, Pitangus sulphuratus e Passer domesticus, sendo que essas espécies se beneficiaram ao consumirem restos de alimentos deixados por visitantes. Altas frequências de ocorrência como apresentadas por essas aves indicam o elevado grau de urbanização das áreas do estudo (FRANCHIN; MARÇAL JR., 2004).

Os registros pouco frequentes de diversas espécies podem ser explicados pelo aparecimento temporário de aves migratórias ou que permaneçam pouco tempo no local (POZZA, 2002), pela vocalização pouco conspícua e/ou baixa densidade populacional na área (ALEIXO; VIELLIARD, 1995). Ainda, espécies ocasionais podem ter nascido em áre- as verdes e acabam se dispersando para outras áreas quando não acham espaço para fixar seu território, havendo um fluxo constante entre as diferentes áreas urbanas (ARGEL-DE-OLIVEIRA, 1995).

Em relação à reprodução, a nidificação de Zenaida auriculata e Furnarius rufus no Parque Tarquínio e de Zenaida auriculata no Parque Paulo Gorski também foi registrada por Model, Remor e Nascimento (2014) no período de dezembro de 2007 a outubro de 2008, confirmando que essas espécies são residentes nos parques. Ninhos de Furnarius rufus observados pelos autores no Parque Paulo Gorski também foram observados neste parque no presente estudo, entretanto, atividades reprodutivas não foram confirmadas.

A presença dos ninhos de C. haemorrhous em uma araucária foi observada durante todo o período da pesquisa. Segundo Feekes (1981) e Duca e Marini (2004), a espécie C. haemorrhous não apresenta preferência por alguma espécie de árvore para realização da nidificação, podendo utilizar outras espécies para formar sua colônia; entretanto, outras espécies de plantas não foram utilizadas para a construção de ninhos neste local. A reprodução em colônias de espécies de Icteríneos parece ser extremamente importante durante o período reprodutivo, pois os machos emitem chamados de alerta com a aproximação de predadores e atacam os intrusos (DUCA; MARINI, 2004; FEEKES, 1981; ROBINSON, 1986). Duca e Marini (2004) observaram ninhos de espécies da família Tyrannidae, sugerindo que haja associação entre estas e C. haemorrhous, devido aos benefícios de uma defesa mais eficaz contra predadores. Porém, neste trabalho não foram registrados ninhos de outras espécies de aves na mesma árvore onde a colônia se estabeleceu.

No que diz respeito à alimentação do tipo nectarivoria no Bosque Elias Lopuch (Tabela 1), C. lucidus ao se alimentar das flores de Jacaranda mimosifolia, atuou como um pilhador de néctar (INOUYE, 1983; MENDONÇA; ANJOS 2005), devido à remoção dos recursos florais oferecidos sem realizar a polinização da planta (MALOOF; INOUYE, 2000), obtendo o néctar através de perfurações na base da corola.

Em relação à frugivoria (Tabela 3), a ingestão de frutos de Nectandra rigida e Ocotea pulchella está relacionada à grande quantidade de energia que elas oferecem, já que as espécies de Lauraceae possuem frutos com uma grande se- 
mente que está envolvida por um pericarpo fino e polpa com alto percentual de lipídios (SNOW, 1981). A observação de apenas duas espécies de aves se alimentando dos frutos de $N$. rigida no Parque Tarquínio (T. rufiventris e T. amaurochalinus) pode estar relacionada à existência de outras plantas frutificando simultaneamente no parque, como Blepharocalyx salicifolius e Ilex paraguariensis, com maior quantidade de espécies de aves se alimentando de seus frutos, podendo ser melhor opção para as aves.

No Parque Danilo Galafassi foi observado um alto consumo de Ficus calyptroceras (Tabela 7). Segundo Ragusa-Netto (2002), um alto consumo desses frutos pelas espécies Tangara sayaca, Turdus amaurochalinus e Pitangus sulphuratus as tornam potenciais dispersoras dessa planta. Essa espécie de figueira produz anualmente abundante quantidade de sementes viáveis, altamente disseminadas pela avifauna (LORENZI, 2002) e, juntamente com outras plantas do gênero Ficus, constitui importante recurso alimentar para as aves (DAVID; MURUGAN; MANAKADAN, 2012; DAVID; MANAKADAN; GANESH, 2015; NILES, 2015), e podem ser um recurso chave para as aves frugívoras em períodos onde há escassez de alimento, pois não apresentam sazonalidade (DAVID; MURUGAN; MANAKADAN, 2012; NISHIDA; NADIE; PAGNIN, 2014). Entretanto, segundo Sick (1997), T. sayaca não é uma espécie considerada boa dispersora de sementes já que os indivíduos mandibulam bagas e as deixam cair sob a planta-mãe.

A planta Cupania vernalis (cujos frutos foram ingeridos por aves no Parque Paulo Gorski e no Bosque Elias Lopuch; Tabela 3) é utilizada em plantios mistos destinados à recuperação de áreas degradadas de preservação permanente e também no paisagismo, tendo grande importância para a fauna, que se beneficiam de seus frutos, principalmente as aves, que são atraídas pela coloração laranja e sabor adocicado do arilo que envolve as sementes (LORENZI, 2000). Indivíduos de $C$. haemorrhous foram observados se alimentando apenas do arilo que envolve as sementes, sendo esta uma espécie com baixo potencial dispersor da planta. As aves estudadas parecem ter um baixo potencial de dispersão de sementes das plantas cujos frutos foram ingeridos, apesar da presença de fatores que contribuam com a dispersão. Visitas curtas à planta diminuem a probabilidade de que sementes ingeridas sejam eliminadas sob a própria planta-mãe e causam um aumento na probabilidade de sua dispersão, mas caso as visitas sejam muito frequentes, esse aumento pode ser perdido (ARGEL-DE-OLIVEIRA, 1999), o que ocorreu com maior frequência no presente estudo. Entretanto, o grande número de visitas sugere que seus frutos constituam um importante recurso alimentar para os dispersores (FRANCISCO; GALETTI, 2002), apresentando uma grande importância ecológica para as aves. É possível que essas aves, ao ingerir o fruto inteiro, como observado em algumas das espécies, possam defecar as sementes longe da planta-mãe, facilitando a dispersão das plantas. Outro fator que aumenta a chance de dispersão da planta é o comportamento de coletar a semente e voar com ela no bico, podendo ser derrubada em locais distantes da planta-mãe (MANHÃES, 2003), porém, no presente estudo, poucas espécies apresentaram esse comportamento.

A maioria das formações vegetais passa por um ou mais períodos de relativa escassez de frutos ao longo do ano que obrigam as aves frugívoras a alterar a dieta ou realizar deslocamentos em busca de novas áreas de alimentação. Exemplos de espécies que realizam deslocamentos são Tyrannus savana e Empidonomus varius, que foram registradas nos meses de setembro a fevereiro nos parques, não sendo registrados nos outros meses devido ao início da sua migração em direção ao norte do Brasil, onde buscam novos recursos alimentares que estarão indisponíveis na região sul nessa época do ano. A presença dessas espécies no ambiente urbano mostra que a vegetação é de grande importância tanto, para aves locais/residentes quanto para aves migratórias e dispersantes (ARGEL-DE-OLIVEIRA, 1995). Muitas espécies de aves frugívoras durante o período de frutificação abundante, geralmente no verão, alteram a dieta para a insetivoria no período de escassez de frutos, como Turdus leucomelas, Turdus rufiventris, Turdus amaurochalinus e Cyanocorax chrysops.

Os resultados do presente trabalho indicam que as aves têm se adaptado ao ambiente urbano, corroborando com os resultados obtidos por Akram, Ilyas e Prusty (2015). Entretanto, apenas os parques maiores e mais complexos conseguem suportar uma maior diversidade de espécies, fornecendo melhores recursos para as aves que frequentam esses locais.

\section{Conclusão}

Registrar como cada espécie faz uso dos recursos do parque é importante para determinar quais recursos podem ser manejados para estimular a permanência e/ou reprodução de uma determinada espécie em parques urbanos como do presente estudo. Verificou-se que estes são importantes para um grande número de espécies de aves, fornecendo recursos alimentares, local para nidificação e descanso, podendo ser utilizados como locais para a conservação das mesmas dentro da área urbana.

Todas as plantas observadas nos parques constituem um importante recurso alimentar para as aves, sejam elas frugívoras, granívoras ou nectarívoras. O manejo nos parques deveria ser feito de modo que plantas nativas da região fossem plantadas nos mesmos, já que, além de importantes para a alimentação das aves, podem ser dispersas pelas mesmas, ter a polinização facilitada e recompor a flora da região.

É importante que se desenvolvam projetos de conservação nos parques para que essas aves continuem utilizando seus recursos. A presença dessas espécies, aliada ao acúmulo de informações sobre elas e suas interações com o parque devem ser explorados como material básico para Educação Ambiental e sensibilização da população da importância de espaços semelhantes.

\section{Agradecimentos}

Agradecemos especialmente à Fundação Araucária pelo apoio financeiro ao projeto de pesquisa, e à Universidade Estadual do Oeste do Paraná por ter cedido materiais importantes para a pesquisa.

\section{Referências}

ADLER, F. R.; TANNER, C. J. Ecossistemas urbanos. São 
Paulo: Oficinas de Textos, 2015. 384 p.

AKRAM, F.; ILYAS, O.; PRUSTY, B. A. Impact of urbanization on bird community structure in and around Aligarh City, U. P., India. International Journal of Engineering Technology Science and Research, v. 2, n. 10, p.2394-3386, 2015.

ALEIXO, A.; VIELLIARD, J. M. E. Composição e dinâmica da avifauna da mata de Santa Genebra, Campinas, São Paulo, Brasil. Revista Brasileira de Zoologia, Curitiba, v.12, n.3, p.493-511, 1995.

ARGEL-DE-OLIVEIRA, M. M. Aves e vegetação em um bairro residencial da cidade de São Paulo (São Paulo, Brasil). Revista Brasileira de Zoologia, Curitiba, v. 12, n. 1, p. 81-92, 1995

ARGEL-DE-OLIVEIRA, M. M.; CASTIGLIONI, G. D. A.; SOUZA, S. B. Comportamento alimentar de aves frugívoras em Trema micrantha (Ulmaceae) em duas áreas alteradas do sudeste brasileiro. Ararajuba, Belém, v. 4, p. 51-55, 1996.

\section{ARGEL-DE-OLIVEIRA, M. M. Frugivoria por aves em um fragmento de floresta de restinga no estado do Espírito Santo, Brasil. Campinas, 1999. 153 f. Tese (Doutorado em Ecologia) - Instituto de Biologia, Universidade de Campinas.}

BARTH, B. J.; FITZGIBBON, S. I.; WILSON, R. S. New urban developments that retain more remnant trees have greater bird diversity. Landscape and Urban Planning, v. 136, p. 122-129, 2015.

CAETANO, T. Frugivoria por aves no Parque Ambiental de Cascavel, Paraná - Brasil. Cascavel, 2005. 37 f. Monografia (Tese de Conclusão de Curso em Ciências Biológicas) - Centro de Ciências Biológicas e da Saúde, Universidade do Oeste do Paraná.

CHATERJEE, S.; BASU, P. Avian frugivory and seed dispersal of a large fruited tree in an Indian moist deciduous forest. Acta Oecologica, v. 65-66, p. 32-40, 2015.

CBRO, Comitê Brasileiro de Registros Ornitológicos.

Listas das aves do Brasil, 11. ed. 1/1/2014. Disponível em: $<$ http://www.cbro.org.br>. Acesso em: 14 mar. 2014.

DAVID, J. P.; MURUGAN, B. S.; MANAKADAN, R. Seasonality in fruiting of fig and non-fig species in a tropical dry evergreen forest in Sriharikota Island, southern India. Tropical Ecology, Varanasi, v. 53, n. 1, p. 1-13, 2012.

DAVID, J. P.; MANAKADAN, R.; GANESH, T. Frugivory and seed dispersal by birds and mammals in the coastal tropical dry evergreen forests of southern India: a review. Tropical Ecology, Varanasi, v. 56, n. 1, p. 41-55, 2015.

DRUMOND, M. A. et al. Sociabilidade das espécies florestais da caatinga em Santa Maria da Boa Vista-PR.

Boletim de Pesquisa Florestal, Colombo, v. 4, p. 47-59,
1982.

FRANCISCO, M. R.; GALETTI, M. Aves como potenciais dispersoras de sementes de Ocotea pulchella Mart. (Lauraceae) numa área de vegetação de cerrado do sudeste brasileiro. Revista Brasileira de Botânica, São Paulo, v. 25, p. 11-17, 2002.

GUIMARÃES, M. Há mais aves nos grandes centros urbanos hoje? Ciência \& Cultura, São Paulo, v. 58, n. 2, p. 14-15, abr./jun. 2006.

IAP, Instituto Ambiental do Paraná. Fauna do Paraná em extinção. Curitiba: Instituto Ambiental do Paraná, 2006. $272 \mathrm{p}$.

INOUYE, D. W. The ecology of nectar robbing. In: Bentley, B. \& Elias, T. The biology of nectaries.New York: Columbia University Press, 1983. p. 153-174.

IUCN. The IUCN red list of threatened species. 2015. Disponível em: <http://www.iucnredlist.org > Acesso em: 02 dez. 2015.

LORENZI, H. Árvores brasileiras:manual de identificação e cultivo de plantas arbóreas nativas do Brasil, v. 1. 3. ed. Nova Odessa: Instituto Plantarum, 2000. $384 \mathrm{p}$.

LORENZI, H. Árvores brasileiras: manual de identificação e cultivo de plantas arbóreas do Brasil, v. 2.2. ed. Nova Odessa: Instituto Plantarum, 2002. 368 p.

MALOOF, J. E.; INOUYE, D. W. Are nectar robbers cheaters or mutualists? Ecology, Washington, v. 81, n. 10, p. 2651-2661, 2000.

MANHÃES, M. A. Dieta de traupíneos (Passeriformes, Emberizidae) no Parque Estadual do Ibitipoca, Minas Gerais, Brasil. Iheringia, Série Zoologia, Porto Alegre, v. 93, p.59-73,2003.

MATARAZZO-NEUBERGER, W. M. Comunidade de cinco parques e praças da Grande São Paulo, estado de São Paulo. Ararajuba, Belém, v. 3, p. 13-19, 1995.

MCKINNEY, M. L. Urbanization, biodiversity and conservation. BioScience, Uberlândia, v. 52, n. 10, p. 883$890,2002$.

MENDONÇA, L.B.; ANJOS, L. Beija-flores (Aves, Trochilidae) e seus recursos florais em uma área urbana do Sul do Brasil. Revista Brasileira de Zoologia, Curitiba, v.22, p. 51-59, 2005.

MENDONÇA-LIMA, A.; FONTANA, C. S. Composição, frequência e aspectos biológicos da avifauna no Porto Alegre Country Clube, Rio Grande do Sul. Ararajuba, Belém, v. 8, n. 1, p. 1-8, 2000.

MODEL, K. J.; REMOR, M. B.; NASCIMENTO, J. E. 
Levantamento qualitativo e reprodutivo da ornitofauna dos parques Tarquínio Joslin dos Santos e Parque Ecológico Paulo Gorski, Cascavel - PR. Arquivos de Ciências

Veterinárias e Zoologia da UNIPAR, Umuarama, v. 17, n. 2, p. 107-114, abr./jun. 2014.

MOTTA-JÚNIOR, J. C. Estrutura trófica e composição das avifaunas de três ambientes terrestres na região central do estado de São Paulo. Ararajuba, Belém, v. 1, p.65-71, 1990.

MUELLER-DOMBOIS, D.; ELLENBERG, H. Aims and methods of vegetation ecology. New York: Wiley, 1974. $547 \mathrm{p}$.

NASON, J. D.; HAMRICK, J. L. Reproductive and genetic consequences of forest fragmentation: two cases studies of neotropical canopy trees. Journal of Heredity, Oxford, v. 88, n. 4, p. 264-276, 1997.

NILES, H. Ficus sp. y la frugivoría: una investigación sobre un recurso importante para las aves en el bosque nublado occidental del Ecuador. Independent Study Project (ISP) Collection, paper 2132, 2015.

NISHIDA, S. M.; NAIDE, S. S.; PAGNIN, D.Plantas que atraem aves e outros bichos. São Paulo: Cultura Acadêmica, 2014. 93 p.

PENTEADO, H. M.; ALVAREZ, C. E. Corredores verdes urbanos: estudo da viabilidade de conexão das áreas verdes de Vitória. Paisagem Ambiente: ensaios, São Paulo, v. 24, p. 57-68, 2007.

PIACENTINI, V. Q. et al. Lista comentada das aves do Brasil pelo Comitê Brasileiro de Registros Ornitológicos. Revista Brasileira de Ornitologia, v. 23, n. 2, p. 91-298, 2015.

PORTAL DO MUNICÍPIO DE CASCAVEL. Bosques. Disponível em: <http:/www.cascavel.pr.gov.br/secretarias/ semdec/sub pagina.php?id=257>. Acesso em: 23 dez. 2017.

PORTAL DO MUNICÍPIO DE CASCAVEL. Parques. Disponível em: <http://www.cascavel.pr.gov.br/secretarias/ semdec/sub_pagina.php?id=258>. Acesso em: 02 dez. 2015.

RAGUSA-NETTO, J. Fruiting phenology and consumption by birds in Ficus calyptroceras (Miq.) Miq. (Moraceae). Brazilian Journal of Biology, São Carlos, v. 62, n. 2, p. 339-346, 2002.

R Core Team. R: A language and environment for statistical computing. 2013. Disponível em: <http://www.r-project. org/>. Acesso em: 23 dez. 2017.

SEMPO, G.; CANONGE, S.; DENEUBOURG, J. L.

From aggregation to dispersion: how habitat fragmentation prevents the emergence of consensual decision making in a group. PLOS ONE, v. 8, n. 11, e78951, 2013.
SICK, H. Ornitologia brasileira. Rio de Janeiro: Nova Fronteira, 1997. 912 p.

SNOW, D. W. Tropical frugivorious birds and their food plants: a world survey. Biotropica, v. 13, p.1-14, 1981.

TILMAN, D. Global environmental impacts of agricultural expansion: The need for sustainable and efficient practices.

Proceedings of the National Academy of Sciences of the

United States of America, v. 96, n. 11, p. 5995-6000, 1999.

VALADÃO, R. M.; FRANCHIN, A. G.; MARÇAL JR, O. A avifauna no Parque Municipal Victório Siquierolli, zona urbana de Uberlândia (MG). Biotemas, Florianópolis, v. 19, p. 81-91, 2006.

VOSS, W. A. Aves de ambientes urbanos. Universidade, v.2, n. 4, 8-9, 1984.
Recebido em: 11.01.2016 Aceito em: 01.03.2018 